\title{
Admission Cardiotocography as a Predictor of Low Apgar Score: An Observational, Cross- Sectional Study
}

\author{
Laila Nazir $^{1}$, Gul Lakhta ${ }^{2}$, Khunsa Anees ${ }^{3}$, Fahad R. Khan ${ }^{4}$, Sabah Safdar ${ }^{1}$, Gul R. Nazir ${ }^{5}$, Mehwish I.
} Irum $^{6}$, Safi U. Khattak ${ }^{4}$, Azra Salim ${ }^{2}$

1. Obstetrics and Gynaecology, Khyber Teaching Hospital, Peshawar, PAK 2. Obstetrics and Gynaecology, Lady Reading Hospital, Peshawar, PAK 3. Paediatrics, Services Institute of Medical Sciences, Lahore, PAK 4. Cardiology, Lady Reading Hospital, Peshawar, PAK 5. Radiology, Khyber Medical University, Peshawar, PAK 6. Radiology, Khyber Teaching Hospital, Peshawar, PAK

Corresponding author: Gul Lakhta, drgullakhta@gmail.com

\section{Abstract}

\section{Background and objective}

Cardiotocography (CTG) has been used more frequently in recent decades to reduce intrapartum fetal mortality rates. The purpose of this study was to determine whether pathological or non-reactive CTG could predict a low Apgar (Appearance, Pulse, Grimace, Activity, and Respiration) score. An abnormal trace would indicate a distressed fetus, whereas a normal trace would indicate a well-oxygenated fetus.

\section{Methods}

This study included a total of 470 women with a gestational period of more than 37 weeks. Based on the results of their CTGs, they were divided into three groups. An emergency cesarean section (CS) was performed if there was any sign of fetal distress on CTG. The Apgar scoring for newborns was recorded in the proforma following delivery.

\section{Results}

The study was carried out at two major tertiary-care hospitals in Pakistan. A reactive CTG was found in more than one-third (39.36\%) of the 470 patients. An Apgar score above 8 was obtained by $34.26 \%$ of the newborns, while an Apgar score below 8 was obtained by more than half (63.40\%). Only $2.34 \%$ of newborns had an Apgar score below 6. A third (30.64\%) of the patients had grade-1 meconium-stained liquor (MSL), $24.89 \%$ had grade-2 MSL, $19.79 \%$ had grade-3 MSL, and $24.68 \%$ had no MSL. One-third (32.34\%) of the neonates were admitted to the neonatal intensive care unit (NICU) shortly after birth. When CTG was pathological or non-reactive, the odds of securing a higher Apgar score decreased by $70.45 \%$ (OR: 0.30; 95\% CI: $0.20-0.44 ; \mathrm{p}<0.001)$.

Review began 04/09/2021 Review ended 04/11/2021 Published 04/17/2021

\section{() Copyright 2021}

Nazir et al. This is an open access article distributed under the terms of the Creative Commons Attribution License CC-BY 4.0., which permits unrestricted use, distribution, and reproduction in any medium, provided the original author and source are credited.

\section{Conclusion}

The main conclusion drawn from this study's findings is that a pathological CTG is an indicator of a low Apgar score.

Categories: Obstetrics/Gynecology, Pediatrics, Quality Improvement

Keywords: cardiotocography, ctg, low apgar score, neonatal mortality, electronic fetal monitoring, maternal and fetal medicine, fetal distress

\section{Introduction}

The surveillance of fetal heart rate is a major element of antenatal care. The purpose of prenatal screening during childbirth is to recognize fetuses at risk of hypoxia during delivery and intervene if required to avoid fetal compromise [1-3]. Prior to the actual invention of the Pinard stethoscope, it was impossible to determine the fetus's condition before birth. The only available option was to rely on the development of the uterus and the mother's awareness of fetal activity. Eventually, the auscultation of fetal heart sounds and the condition of amniotic fluid were used to determine the fetus's state during childbirth. Similarly, meconium-stained liquor (MSL) had been considered a typical sign of fetal distress.

Since its advent, cardiotocography (CTG) or intrapartum electronic fetal monitoring (EFM) has become a modern functional tool for fetal monitoring during pregnancy and delivery [4]. It measures fetal heart rate pattern and its relationship with uterine contractions [3]. CTG provides details regarding the fetal state. Its usage has gradually expanded over the past couple of decades and has contributed significantly to decreasing intrapartum fetal death rates [2]. With the emergence of modern technologies of EFM, there is a likelihood of lowering neonatal deaths due to hypoxia by $60 \%$ [2]. A normal trace is a sign of a well-oxygenated fetus, 
but an abnormal trace implies a distressed fetus [2]. It is important to note that CTG recording can neither act as a substitute for clinical expertise nor an excuse to leave the patient unsupervised during childbirth. Cesarean and instrumental deliveries have been on the rise over the last four decades [5]. CTG is a major contributor to the rise of operational deliveries. Yet, studies have reported incidences of false-positive CTG traces that had caused unnecessary operational deliveries [6]. CTG has a positive predictive value of $60 \%$ for fetal distress [6]. Besides its role in the rise in operational deliveries, CTG or EFM is considered superior to intermittent auscultation [7]. However, according to Beard et al., CTG is a subjective test; therefore, it alone should not be relied upon to avoid unnecessary interventions [8].

The regular use of admission CTG in low-risk pregnancies has been linked to a rise in operational deliveries with no decline in perinatal mortality [9]. Some health professionals have proposed that CTG should be performed only in cases with a strong probability of potential fetal hypoxia/acidosis related to maternal, medical, or fetal problems. This issue of risk versus benefits related to admission CTG has created a gap in the literature. Our primary objective in carrying out this study was to fill this gap, keeping in view the limitations of CTG and to prove the authenticity of admission CTG and its role in predicting fetal distress [taking low Apgar (Appearance, Pulse, Grimace, Activity, and Respiration) scores as a measure of fetal distress]. This study aimed to evaluate the role of pathological or non-reactive CTG in predicting low Apgar scores.

\section{Materials And Methods}

We conducted a cross-sectional, observational study in the Khyber Teaching Hospital (KTH) and Lady Reading Hospital (LRH) in Peshawar, Pakistan from April 1, 2020, to September 30, 2020. These two hospitals are the main tertiary-care centers in the Khyber Pakhtunkhwa state of Pakistan. The study enrolled 470 patients with a gestational period of over 37 weeks. Non-probability consecutive random sampling was used as the sampling method.

The approval from the Ethical Review Committees and informed consent from patients were obtained prior to the commencement of the study. Admission CTGs were carried out in the participants. Patients were categorized into three groups based on the results of their CTGs: (1) patients with reactive CTGs, (2) patients with non-reactive CTGs, and (3) patients with suspicious CTGs, as per the guidelines issued by the National Institute of Clinical Excellence (NICE) [10]. These CTGs varied based on the heart rate pattern and the absence or presence of acceleration, variability, and deceleration. MSL was recorded and graded accordingly. Grade-1 MSL was translucent and light yellow-green in color, grade-2 MSL was opalescent with deep green and light yellow in color, and grade- 3 was opaque and deep green in color.

Upon any sign of fetal distress on CTG (i.e., baseline fetal heart rate below 110 or above 160 along with loss of variability or late decelerations), an emergency cesarean section (CS) was performed. After delivery, the newborn's Apgar score was recorded at five minutes in the patient's proforma along with other demographic details.

\section{Inclusion criteria}

We only enrolled those patients who met the eligibility criteria, which were as follows: gestational ages ranging from 37 to 42 weeks, ages ranging from 18 to 40 years, and both primigravida and multigravida.

\section{Exclusion criteria}

Patients with multiple pregnancies, known fetal anomalies, gestational diabetes, and pre-eclampsia were excluded to reduce the confounding effect. Fetuses with Cord-Around-the Neck (CAN; diagnosed on ultrasonography) were excluded as well.

\section{Data analysis}

Data were analyzed using SPSS Statistics version 25.0 (IBM, Armonk, NY). We presented continuous variables as mean \pm standard deviation, while categorical variables were presented as frequencies and percentages. An independent t-test was conducted to assess if there were significant differences in age and BMI between the CTG results. A chi-square test was carried out for categorical variables, taking a p-value of $\leqslant 0.05$ as significant. After controlling for age and BMI, we used an ordinal logistic regression analysis to see if differences in CTG results could explain the odds of newborns having different Apgar scores.

\section{Results}

This study was conducted at the Obstetrics and Gynecology departments of two tertiary-care hospitals in Pakistan, with a view to determining the frequency of low Apgar scores in pregnant women with abnormal CTG findings. The age range of the patients was $23-40$ years, and the mean patient age was 30.19 years ( $\mathrm{SD}=5.73, \mathrm{Mdn}=30.00) ; 203$ patients (43.19\%) were between $15-30$ years, while 267 (56.81\%) were between 31-45 years of age. Our sample's BMI was in the range of $20.75-35 \mathrm{~kg} / \mathrm{m}^{2}$, and the mean BMI was 27.04 $(\mathrm{SD}=5.28, \mathrm{Mdn}=23.18)$. Of the total 470 patients, 185 (39.36\%) had a normal or reactive CTG, 28 (5.96\%) 


\section{Cureus}

patients had a suspicious CTG, while 257 (54.68\%) patients exhibited a pathological or non-reactive CTG. Among the 470 patients, 225 (47.77\%) had a cesarean delivery, while 246 (52.23\%) had a normal vaginal delivery. The Apgar score at five minutes of delivery was calculated; 161 (34.26\%) of the newborns had an Apgar score above 8, 298 (63.40\%) had an Apgar score below 8, and 11 (2.34\%) of the newborns had an Apgar score below 6. Among the total participants, grade-1 MSL was found in 144 (30.64\%) patients; 117 (24.89\%) had grade-2 MSL, 93 (19.79\%) had grade-3 MSL, and no MSL was noted in 116 (24.68\%) patients. Upon delivery, 152 (32.34\%) of the newborns were admitted to the neonatal Intensive care unit (NICU). These results are presented in Table 1 .

\begin{tabular}{|c|c|c|c|c|c|}
\hline Variable & Mean & SD & Mdn & $\mathbf{N}$ & $\%$ \\
\hline Age, years & 30.19 & 5.73 & 30 & & \\
\hline Body mass index (BMI), $\mathrm{kg} / \mathrm{m}^{2}$ & 27.04 & 5.28 & 23.18 & & \\
\hline \multicolumn{6}{|l|}{ Age group } \\
\hline $15-30$ years & & & & 203 & 43.19 \\
\hline $31-40$ years & & & & 267 & 56.81 \\
\hline \multicolumn{6}{|l|}{ Cardiotocography (CTG) } \\
\hline Reactive/normal & & & & 185 & 39.36 \\
\hline Suspicious & & & & 28 & 5.96 \\
\hline Pathological/non-reactive & & & & 257 & 54.68 \\
\hline \multicolumn{6}{|l|}{ Apgar score } \\
\hline$>8$ & & & & 161 & 34.26 \\
\hline$<8$ & & & & 298 & 63.4 \\
\hline$<6$ & & & & 11 & 2.34 \\
\hline \multicolumn{6}{|l|}{ Meconium-stained liquor (MSL) } \\
\hline None & & & & 116 & 24.68 \\
\hline Grade-1 & & & & 144 & 30.64 \\
\hline Grade-2 & & & & 117 & 24.89 \\
\hline Grade-3 & & & & 93 & 19.79 \\
\hline \multicolumn{6}{|c|}{ Neonatal intensive care unit (NICU) } \\
\hline Yes & & & & 152 & 32.34 \\
\hline No & & & & 318 & 67.66 \\
\hline
\end{tabular}

\section{TABLE 1: Summary of all the categorical and continuous variables used in the study}

SD: standard deviation; Mdn: median; Apgar: Appearance, Pulse, Grimace, Activity, and Respiration

We then compared continuous and categorical variables among the subgroups of CTG using t-test and chisquare test, as shown in Table 2 and Table 3. 


\section{Cureus}

\begin{tabular}{|c|c|c|c|c|c|}
\hline Variable & Mean & SD & $\mathbf{N}$ & Mdn & P-value \\
\hline Age, years & & & & & 0.003 \\
\hline Reactive/normal & 29.097 & 5.771 & 185 & 26.000 & \\
\hline Suspicious & 30.071 & 5.843 & 28 & 30.500 & \\
\hline Pathological/non-reactive & 30.996 & 5.572 & 257 & 32.000 & \\
\hline Body mass index, kg/m² & & & & & 0.071 \\
\hline Reactive/normal & 26.928 & 5.308 & 185 & 23.183 & \\
\hline Suspicious & 24.967 & 5.237 & 28 & 22.161 & \\
\hline Pathological/non-reactive & 27.352 & 5.239 & 257 & 23.183 & \\
\hline
\end{tabular}

TABLE 2: Comparison of the continuous variables among the subgroups of CTG using t-test SD: standard deviation; Mdn: median; CTG: cardiotocography

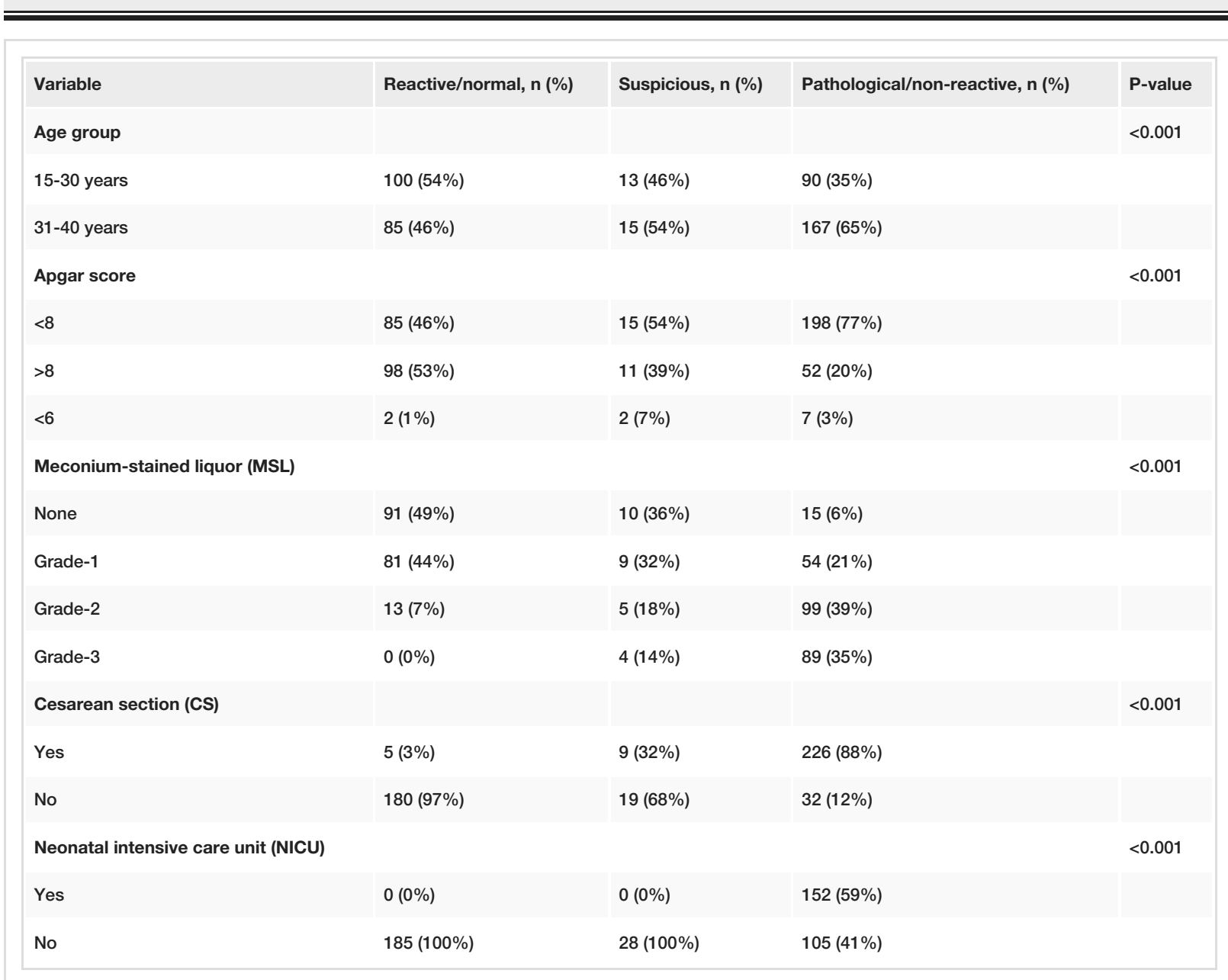

TABLE 3: Comparison of the categorical variables among the subgroups of CTG using chi-square test

Apgar: Appearance, Pulse, Grimace, Activity, and Respiration; CTG: cardiotocography

The regression coefficient for age, BMI, and suspicious CTG were not significant, suggesting that they did not affect the Apgar score. The regression coefficient for non-reactive CTG was significant, indicating that 
the odds of observing a higher Apgar score decreased by 70.45\% when CTG was pathological or non-reactive (OR: $0.30 ; 95 \%$ CI: $0.20-0.44 ; \mathrm{p}<0.001$ ). The results of the ordinal logistic regression analysis are shown in Table 4.

\begin{tabular}{|c|c|c|c|c|c|c|}
\hline Predictor & B & SE & $x^{2}$ & P-value & OR & $95 \% \mathrm{Cl}$ \\
\hline Age & -0.01 & 0.02 & 0.2 & 0.657 & 0.99 & $0.96-1.03$ \\
\hline Body mass index (BMI) & 0 & 0.02 & 0.03 & 0.87 & 1 & $0.97-1.04$ \\
\hline Suspicious CTG & -0.13 & 0.4 & 0.11 & 0.741 & 0.88 & $0.40-1.92$ \\
\hline Pathological/non-reactive CTG & -1.22 & 0.21 & 34.19 & $<0.001$ & 0.3 & $0.20-0.44$ \\
\hline
\end{tabular}

TABLE 4: Ordinal logistic regression results for age, BMI, and CTG as predictors of Apgar score

B: unstandardized beta; SE: standard error; OR: odds ratio; $\mathrm{X}^{2}$ : chi-square test statistic; $\mathrm{Cl}$ : confidence interval; BMI: body mass index; CTG: cardiotocography; Apgar: Appearance, Pulse, Grimace, Activity, and Respiration

\section{Discussion}

In this study, we analyzed the link between admission CTG during labor and its implications on perinatal morbidity and mortality. EFM has been instrumental in decreasing perinatal mortality and illness. We discovered that pathological CTG is associated with a low Apgar score and NICU admissions. The mean age in our study group was $30.21 \pm 5.73$ years, while most patients were in the age group of 31-45 years. Though most of our patients were not overweight, our sample's mean BMI was $27 \pm 5.28 \mathrm{~kg} / \mathrm{m}^{2}$. Of the total 470 patients, more than a quarter had a normal or reactive CTG; a small proportion had a suspicious CTG, while more than half of the patients exhibited a pathological or non-reactive CTG. Apgar score at five minutes of delivery was calculated. A third of the neonates had an Apgar score above 8, two-third had an Apgar score below 8 but above 6, and only 2\% had an Apgar score below 6.

Chaudhari et al. reported reactive CTGs in $74 \%$ of their study sample, suspicious CTGs in $16 \%$, and pathological traces in 10\% [9]. On the contrary, in our study, 39\% had reactive CTG, 6\% had suspicious CTG, and 55\% had a pathological CTG. In their study on 200 low-risk patients, Hegde et al. reported that fetal distress was linked to suspected and pathological CTG on admission. Therefore, the frequency of operational deliveries also continued to increase [11]. These findings were consistent with our findings.

Das et al. carried out a study to determine the usefulness of the CTG at admission in forecasting the risk of fetal distress during labor. The frequency of fetal distress and operational delivery was relatively high in the non-reassuring CTG group [12]. In our study, none of the patients with reassuring CTGs had their neonates admitted to NICU. Only two patients out of 470 had babies with an Apgar score below 6, despite a normal CTG trace. These findings favor the authenticity of admission CTG.

Furthermore, a study done by Rahman et al. reached findings similar to our study. Out of the total patients with reactive CTG, almost $10 \%$ of patients had MSL, and 6.5\% were admitted to NICU. MSL and NICU admissions were higher by nearly fourfold in the suspicious group compared to patients with reactive CTG. Simultaneously, there was a tenfold increase in MSL and low Apgar score in the pathological group [13]. In our study, $7 \%$ of patients with reactive CTG had grade-2 MSL, which increased to $18 \%$ in patients with suspicious traces and $39 \%$ in patients with pathological traces.

According to a study by Gupta et al., CTG is not a good index of fetal distress; despite reassuring CTG, 34.6\% of infants were born with MSL, one-third had an Apgar score below 7, and around $20 \%$ were admitted to the NICU [14]. Our study contradicted Gupta et al.'s findings because all of our patients admitted to NICU had a pathological CTG on admission. Moreover, our study endorsed findings by Panda et al. In their study, patients with reassuring CTG had reduced MSL rates (4.65\%); only 3.48\% had an Apgar score below 7, and 9.3\% had NICU admissions. While patients in whom CTG was not reassuring, MSL increased dramatically to 85.71\%, one-third had a low Apgar score, and 78.57\% were admitted to NICU [15]. In our study, the percentages of grade-2 MSL, Apgar scores below 8, and NICU admission in cases with reactive CTGs were $7 \%, 46 \%$, and $0 \%$ compared to $39 \%, 77 \%$, and $59 \%$ in non-reactive CTG.

Mires et al. reported false-positive traces in $21.8 \%$ of patients who underwent operational delivery; among them, only $3.6 \%$ were non-reactive [6]. While in our sample, $20 \%$ had a non-reactive CTG, but the Apgar score above 8 . Impey et al. demonstrated one-third of the traces to be non-reactive [16]. While in our study, more than half of the CTG traces were non-reactive. 
In the study by Kansal et al., fetal distress was present in 16\% of patients with normal admission CTG, 63\% with suspicious CTG, and 97\% with pathological CTG [17]. In comparison, fetal distress was seen in $46 \%$, $54 \%$, and $77 \%$ of our sample's normal, suspicious, and pathological CTGs respectively. Bix et al. analyzed three trials and found that the admission CTG was not effective in predicting neonatal outcomes [18]. However, our study contradicts the statement of Blix et al. because our research concluded that a pathological CTG trace at admission could be a predictor of a low Apgar score (OR: 0.30; 95\% CI: 0.20-0.44; $\mathrm{p}<0.001)$.

Multiple studies have endorsed that CTG plays a decisive role in detecting fetal distress and works as a harbinger of poor neonatal outcomes. A few among those studies are as follows: a study by Akhavan et al. conducted on 425 patients, a study by Sunitha et al. conducted on 100 patients, and the study by Kumar et al. conducted on 320 patients in 2019 [19-22]. Our study also backed the findings of these studies.

Gourounti et al. carried out a meta-analysis of multiple randomized controlled trials (RCTs) and determined that the admission CTG was connected to a rise in operational deliveries [22]. In contrast, in our study, only three patients with reactive CTG traces and six patients with suspicious traces underwent a CS. Larger randomized controlled trials are required to provide more insight into the use of CTG to predict neonatal outcomes and reduce perinatal mortality.

\section{Limitations}

Our study has several limitations. Firstly, we did not focus on the primary cause of a suspicious or pathological CTG, its reversibility, or the delivery timing to avoid excessive fetal hypoxia/acidosis and unnecessary obstetric intervention. Secondly, no additional testing was performed to evaluate fetal oxygenation. Moreover, our study was limited to two hospitals; hence, larger randomized trials are needed to back our findings in this part of the world.

\section{Conclusions}

The CTG is an easy, painless, and cost-effective test for evaluating fetal well-being. It could be used as an effective screening investigation. This test can identify patients who are at high risk for operational delivery. Our research focused on the importance of CTG in detecting fetal distress. The study's prediction of a low Apgar score in cases of a non-reactive CTG trace illustrated the significance of CTG in detecting fetal distress. Our study's findings can prove instrumental in decreasing the need for continuous CTG monitoring or other invasive testing required to detect fetal distress, thereby saving time and resources and mitigating patient discomfort, particularly in those hospitals with a high flow of patients.

\section{Additional Information \\ Disclosures}

Human subjects: Consent was obtained or waived by all participants in this study. Ethical Review Board, MTI/LRH issued approval N/A. This study was approved by the Ethical Review Board, MTI/LRH. Animal subjects: All authors have confirmed that this study did not involve animal subjects or tissue. Conflicts of interest: In compliance with the ICMJE uniform disclosure form, all authors declare the following: Payment/services info: All authors have declared that no financial support was received from any organization for the submitted work. Financial relationships: All authors have declared that they have no financial relationships at present or within the previous three years with any organizations that might have an interest in the submitted work. Other relationships: All authors have declared that there are no other relationships or activities that could appear to have influenced the submitted work.

\section{References}

1. Alfirevic Z, Devane D, Gyte GM, Cuthbert A: Continuous cardiotocography (CTG) as a form of electronic fetal monitoring (EFM) for fetal assessment during labour. Cochrane Database Syst Rev. 2017, 2:CD006066. 10.1002/14651858.CD006066.pub3

2. Vintzileos AM, Nochimson DJ, Guzman ER, Knuppel RA, Lake M, Schifrin BS: Intrapartum electronic fetal heart rate monitoring versus intermittent auscultation: a meta-analysis. Obstet Gynecol. 1995, 85:149-55. 10.1016/0029-7844(94)00320-d

3. Royal College of Obstetricians and Gynaecologists. The use of electronic fetal monitoring. Evidence-based clinical guideline, number 8. (2001). Accessed: March 1, 2021: http://ctgutbildning.se/images/Referenser/RCOG-2001.pdf.

4. Harrington DI, Redman CW, Moulden M, Greenwood CE: The long-term outcome in surviving infants with Apgar zero at 10 minutes: a systematic review of the literature and hospital-based cohort. Am J Obstet Gynecol. 2007, 196:463.e1-5. 10.1016/j.ajog.2006.10.877

5. Clark SL, Hankins GD: Temporal and demographic trends in cerebral palsy--fact and fiction . Am J Obstet Gynecol. 2003, 188:628-33. 10.1067/mob.2003.204

6. Mires G, Williams F, Howie P: Randomised controlled trial of cardiotocography versus Doppler auscultation of fetal heart at admission in labour in low risk obstetric population. BMJ. 2001, 322:1457-60. 10.1136/bmj.322.7300.1457

7. Schifrin BS, Amsel J, Burdorf G: The accuracy of auscultatory detection of fetal cardiac decelerations: a 
computer simulation. Am J Obstet Gynecol. 1992, 166:566-76. 10.1016/0002-9378(92)91674-y

8. Beard RW, Filshie GM, Knight CA, Roberts GM: The significance of the changes in the continuous fetal heart rate in the first stage of labour. J Obstet Gynaecol Br Commonw. 1971, 78:865-81. 10.1111/j.14710528.1971.tb00198.x

9. Chaudhari KR, Pai SS: Admission test for screening labor. Hyderabad in: A comparative study by Dr. Reddy's Laboratories. (2009). Accessed: April 16, 2021: https://scholar.google.com/scholar_lookup? title=Admission\%20test\%20for\%20screening\%20labor \&publication_year=2009\&auth....

10. Wee MY, Brown H, Reynolds F: The National Institute of Clinical Excellence (NICE) guidelines for caesarean sections: implications for the anaesthetist. Int J Obstet Anesth. 2005, 14:147-58. 10.1016/j.ijoa.2004.09.008

11. Hegde A, Kore S, Srikrishna S, Ambiye VR, Vaidya PR: Admission test: screening test for prediction of fetal outcome in labour. J Obstet Gynecol Ind. 2001, 51:40-3.

12. Das V, Katiyar N, Malik GK: Role of admission test. J Obstet Gynecol Ind. 2001, 51:48-50.

13. Rahman H, Prachi R, Sudip D: Reliability of admission cardiotocography in predicting adverse perinatal outcome in low-risk obstetric population. Indian Obstet Gynaecol. 2012, 2:6-12.

14. Gupta M, Nagar T, Gupta P: Role of cardiotocography to improve perinatal outcome in high risk pregnancy . Int J of Contemp Med Res. 2017, 4:853-6.

15. Panda S, Das A, Nowroz HM, Singh AS: Role of admission tests in predicting perinatal outcome: a prospective study. J Preg Child Health. 2015, 2:171. 10.4172/2376-127X.1000171

16. Impey L, Reynolds M, MacQuillan K, Gates S, Murphy J, Sheil O: Admission cardiotocography: a randomised controlled trial. Lancet. 2003, 361:465-70. 10.1016/S0140-6736(03)12464-6

17. Kansal R, Goel G, Mangala D, Garg P, Verma K, Geetika: Correlation of admission test with neonatal outcome. People's J Sci Res. 2014, 7:27-31.

18. Bix E, Reiner LM, Klovning A, Oian P: Prognostic value of the labour admission test and its effectiveness compared with auscultation only: a systematic review. BJOG. 2005, 112:1595-604. 10.1111/j.14710528.2005.00766.x

19. Akhavan S, Lak P, Rahimi-Sharbaf F, Mohammadi SR, Shirazi M: Admission test and pregnancy outcome. Iran J Med Sci. 2017, 42:362-8.

20. Sunitha C, Rao PS, Prajwal S, Bhat RK: Correlation of intrapartum electronic fetal monitoring with neonatal outcome. Int J Reprod Contracept Obstet Gynecol. 2017, 6:2174-79. 10.18203/2320-1770.ijrcog20172299

21. Kumar A, Jaju PB: Admission test cardiotocography in labour as a predictor of foetal outcome in high risk pregnancies. Int J Reprod Contracept Obstet Gynecol. 2019, 8:1331-6. 10.18203/2320-1770.ijrcog20190981

22. Gourounti K, Sandall J: Admission cardiotocography versus intermittent auscultation of fetal heart rate: effects on neonatal Apgar score, on the rate of caesarean sections and on the rate of instrumental delivery-a systematic review. Int J Nurs Stud. 2007, 44:1029-35. 10.1016/j.ijnurstu.2006.06.002 